rev.relac.int.estrateg.segur.9(2):103-118,2014

\title{
LAS TRANSFORMACIONES SOCIALES: UNA DISCUSIÓN SOBRE EL CUERPO, LA FIGURA ESTATAL Y LA INCLUSIÓN/EXCLUSIÓN
}

\author{
Valeria F. Falleti**
}

\section{RESUMEN}

En tiempos de globalización, de crecimientos acelerados producto de las ofertas de la tecnología, de espacios y tiempos impersonales signados, más bien, por la importancia del procedimiento, por la búsqueda de la eficacia y la agilidad, que por la experiencia de los mismos, consideramos relevante realizar una reflexión sobre ciertas categorías y procesos centrales para pensar tanto aspectos sociales como relativos a la producción de subjetividad. A partir de la propuesta de Deleuze del traspaso de las sociedades disciplinarias a las de control, el objetivo de este artículo es analizar las

Este artículo es producto de la discusión realizada en un seminario teórico sobre "Problemas Sociales Contemporáneos", del que participé hace algunos años en la Facultad Latinoamericana en Ciencias Sociales FLACSO, sede México. Algunas de las líneas teóricas aquí plasmadas sirvieron para la argumentación de ciertos aspectos desarrollados en mi tesis doctoral y en el libro que publiqué posteriormente en julio de 2012 (Falleti, 2012).

** Profesora-Investigadora del Departamento de Educación y Comunicación de la División de Ciencias Sociales y Humanidades de la Universidad Autónoma Metropolitana, sede Xochimilco. Correos electrónicos: valeriafalleti@gmail.com; vfalleti@correo.xoc.uam.mx. 
transformaciones en las categorías de cuerpo, estado e inclusión/exclusión. Realizamos una discusión sobre el cuerpo con las conceptualizaciones de discurso, saber y poder de Foucault, para contraponerlas a "el cuerpo sin órganos" de Deleuze. Los cambios en la figura estatal son trabajados con Hardt y Negri. Por su parte, Luhmann nos permite pensar los mecanismos de inclusión y exclusión en las sociedades actuales. Nuestra labor fue la de articular los distintos aportes en función de pensar las particularidades en los procesos sociales y la subjetividad.

Palabras clave: subjetividad, cuerpo, inclusión/exclusión, procesos sociales.

\title{
SOCIAL TRANSFORMATIONS: A DISCUSSION ON THE BODY, THE STATE FIGURE AND INCLUSION/EXCLUSION
}

\begin{abstract}
In times of globalization, of increasing growth as a result of new technologies, of impersonal time and space defined for the importance of the processes and for pursue of efficacy and agility, rather than for experiencing these time and space, it is in this context where we consider relevant to think about some categories and processes that are central to understand both social aspects and others related to the production of subjectivity. Taking into account the proclaimed transition from disciplinary to control societies proposed by Gilles Deleuze, the goal of this article is to consider the transformations in the body, in the state and in the inclusion/exclusion mechanisms. We discuss the notion of body in relation to the concepts of discourse, knowledge and power by Foucault in order to compare them with the idea of "body without organs" by Deleuze. The changes in the notion of state are considered using Hardt and Negri. On the other hand, the work by Luhmann allows us to think about the mechanisms of inclusion and exclusion in contemporary societies. Our task was to articulate these different contributions in order to think about the particularities in the social processes and the subjectivity.
\end{abstract}

Keywords: subjectivity, body, inclusion/exclusion, social, ocesses.

\section{TRANSFORMAÇÕES SOCIAIS: UMA DISCUSSÃO SOBRE O CORPO, A FIGURA ESTATAL E A INCLUSÃO / EXCLUSÃO.}

\section{RESUMO}

Em tempos de globalização, de crescimento acelerado, fruto das ofertas da tecnologia, de espaços e tempos impessoais rubricados, e na verdade, pela importância do processo, pela busca da eficiência e agilidade, que pela própria experiência, consideramos importante fazer uma reflexão sobre certas categorias e processos centrais para pensar tantos aspectos sociais como os relativos à produção de subjetividade. A partir da proposta de Deleuze sobre a transferência de 
sociedades disciplinarias para as de controle, o objetivo deste trabalho é analisar as mudanças nas categorias de corpo, estado e de inclusão/exclusão. Realizamos uma discussão sobre o corpo com as conceituações de discurso, conhecimento e poder de Foucault, para contrapô-las a "o corpo sem órgãos" de Deleuze. As mudanças na figura estatal são trabalhadas com Hardt e Negri. Por sua vez, Luhmann nos permite pensar nos mecanismos de inclusão e exclusão nas sociedades contemporâneas. Nossa tarefa foi a de articular as diferentes contribuições para pensar sobre as particularidades nos processos sociais e na subjetividade.

Palavras-chave: subjetividade, corpo, inclusão / exclusão, processos sociais.

\section{INTRODUCCIÓN 1}

En el presente artículo nos interesa identificary analizar los cambios referidos entre la modernidad y la llamada posmodernidad, o bien dar cuenta del traspaso de las sociedades disciplinarias a las de control ${ }^{2}$. Estas transformaciones se centran en tres aspectos: en la idea de cuerpo, en las modificaciones ocurridas en torno al Estado y en los mecanismos de inclusión-exclusión. Este trabajo explora dimensiones que permiten indagar las transformaciones mencionadas, y contactar diferentes niveles de análisis, una mirada micro con otra macro (Alexander, 1994).

En el primer apartado trabajamos con la noción de cuerpo en Foucault para ponerla en discusión con el aporte de Deleuze en esta temática. En el primer caso, sostenemos que se plantea un cuerpo pasivo capturado y atravesado por el discurso, mientras que el segundo autor propone una idea de cuerpo compuesto por fuerzas activas y reactivas con capacidad para resistir (Lash, 1997). En relación a los cambios en la figura estatal, mostramos la manera en que se pasa de

1. Si bien este artículo se organiza a partir de sostener un traspaso de las sociedades disciplinarias a las de control, o bien de la modernidad a la posmodernidad, no es la intención plantear un cambio radical y absoluto entre un tipo de sociedad y el otro, somos conscientes de que estas distintas lógicas y formas sociales pueden convivir en una misma sociedad. Según las sociedades y las prácticas sociales puede prevalecer una lógica más que otra. Estas distinciones y traspasos sociales los planteamos, sobre todo, con fines expositivos y explicativos.

2. Son distintas las maneras en las que los diferentes autores explican las transformaciones entre modernidad y posmodernidad y, por tanto, las dimensiones de análisis que se relevan. Scott Lash (1997) analiza dichos cambios en términos procesuales y como regímenes de significación que producen objetos culturales en los que interesará su circulación y su modo específico de significación. Este autor propone realizar una genealogía del cuerpo y el deseo, cuestiones que se trabajan en el siguiente apartado. Baumann (1999) analiza las transformaciones en el tiempo, el territorio y el Estado, y ofrece aportes interesantes para pensar los mecanismos de inclusión-exclusión. Negri y Hardt (2002) analizan el Imperio a través de las transformaciones de las figuras jurídicas, el derecho y la autoridad en tanto constitución material del poder y el orden mundiales. El aspecto común entre estos autores es que introducen una dimensión biopolítica y ofrecen una perspectiva dinámica y socio-histórica de estos cambios. Estos autores realizan planteos que van más allá de las visiones que parten de la existencia de procesos globales sin rastrear su procedencia y funcionalidad, y cuyos análisis se limitan a los cambios observados a nivel institucional ya sea de la política o de la economía. 
un poder político sustentado en la razón de Estado a una figura supranacional, por lo tanto, de una soberanía de los Estados-nación a una soberanía supranacional (Negri y Hardt, 2002). Finalmente, en relación a los mecanismos de inclusión-exclusión: mientras en las sociedades disciplinarias la inclusión se constituye en el principio ordenador de la sociedad, en las de control (o posmodernidad) la exclusión es la que cumple esa función (Luhmann, 1998).

Los distintos aspectos analizados se encuentran íntimamente relacionados, dado que las modalidades en que se presentan los mecanismos de inclusión-exclusión responden a maneras específicas de operación e intervención por parte del Estado -en tanto materialización del poder político-y, al mismo tiempo, estos mecanismos y operaciones son coherentes con cierta configuración de cuerpo.

\section{EL CUERPO PASIVO Y LA SALIDA EN EL DESEO DELEUZEANO}

Michel Foucault, en Vigilar y Castigar, plantea que la transición de la edad clásica a la moderna se caracteriza por un "trato" distinto del cuerpo: de castigarlo mediante una acción directa, y en tanto organismo biológico -cuestión explicada a través de la descripción de un suplicio que abre el primer capítulo del libro-, se pasa a castigar algo incorpóreo e intangible como el alma. El alma es una pieza en el dominio que el poder ejerce sobre el cuerpo, efecto e instrumento de una anatomía política y, en este sentido, el alma es prisión del cuerpo. Dice el autor:

"La historia de esta "microfísica" del poder punitivo sería entonces una genealogía o una pieza para una genealogía del "alma" moderna. Más que ver en esta alma los restos reactivados de una ideología, se reconoce en ella más bien el correlato actual de cierta tecnología del poder sobre el cuerpo (...) Pero sí que existe, que tiene una realidad que está producida permanentemente en torno, en la superficie y en el interior del cuerpo por el funcionamiento de un poder que se ejerce sobre aquellos a quienes se vigila, castiga, se educa y corrige. Sobre los locos, los niños, los colegiales, los colonizados, sobre aquellos que se sujetan a un aparato de producción...". (Foucault, 1976, p.36)

Mientras en la concepción clásica de cuerpo este se ofrece como espectáculo para el público, dirigida a la reproducción del poder absolutista (Foucault, 1976; Lash, 1997, p.84), en la concepción moderna se desarrollan dispositivos sofisticados y complejos tales como las instituciones y los saberes, como por ejemplo, la justicia que pretende separarse de la crueldad del verdugo y el saber psiquiátrico que legitima a las acciones punitivas dándoles fuerza de racionalidad. En este sentido, ya no se opera sobre el cuerpo a través de una inscripción física directa sino que este es mediado por saberes, discursos e instituciones que, al mismo tiempo que lo vigilan y controlan, corrigen y manipulan, producen cuerpos útiles y dóciles. Este aspecto productivo del cuerpo se vuelve funcional al vertiginoso desarrollo del capitalismo. 
En el estudio de esta transición a través de la mirada médica, Foucault sostiene que los textos médicos clásicos se atrincheraban en la filosofía de la representación, las inferencias eran deductivas e imperaba el estudio de la anatomía, echando luz sobre la superficie del cuerpo. En la modernidad, con la mirada clínica se llega a conocer al cuerpo y a los órganos "en sí mismos". Se observa un reemplazo de la deducción y la contemplación del cuerpo a una experimentación con el mismo, por lo que podría ser regulado y sus movimientos calculados (Lash, 1997, p.83).

Scott Lash observa que Foucault conceptualiza un cuerpo pasivo, dado que es actuado en escenarios institucionales constituidos discursivamente y no se muestra de qué manera el cuerpo puede construir resistencias y emprender luchas. Es decir, si el discurso es vehículo de poder y el cuerpo queda tematizado a nivel del discurso: cómo sería posible resistir. La pasividad corporal se condice con una visión pesimista del obrar (Lash, 1997: 85). En este sentido Lash, quien pretende establecer un concepto más amplio del "obrar" de la genealogía, sostiene que el trabajo genealógico de Foucault queda incompleto. El autor sostiene: "El problema es que Foucault, a diferencia de Deleuze, opera sin una noción desarrollada de deseo o su equivalente. Por tanto, el cuerpo de Foucault es sólo presa de fuerzas reactivas -normalizadoras e individualizantes- y la genealogía de Foucault queda incompleta" (Lash, 1997, p.92).

Coincidimos en parte con esta crítica dado que, si bien no se desprende una fuerza activa en su concepción de cuerpo, tal como propone Deleuze con su teoría del deseo, consideramos que el aporte valioso de Foucault reside en mostrarnos una lógica que rige en las instituciones modernas que se caracteriza justamente por su carácter reactivo y normalizador de los sujetos. Por esto, se hace referencia a las sociedades disciplinarias. Entonces, más que aludir a un trabajo incompleto, plantearíamos que la genealogía de cuerpo propuesta por Foucault responde a modos de subjetivación propios de las sociedades modernas. Ahora bien, es cierto que estas categorías resultan limitadas para pensar una idea de cuerpo en la posmodernidad. Para esto, la idea de "cuerpo sin órganos", de Deleuze, ofrece pistas interesantes.

Lash rescata de Deleuze su manera de pensar la diferencia y su teoría de deseo, estas nociones nos permiten pensar un cuerpo activo, con intensidades y, por tanto, que resiste; un cuerpo que se inscribe más allá del orden simbólico. En este sentido, sostiene que viene a completar la genealogía incompleta de Foucault, dice: "el Anti-Edipo es parte integrante de la empresa genealógica en su conjunto" (Lash, 1997, p.93).

La concepción deleuzeana de "diferencia" produce una ruptura con una tradición que la ha concebido como una diferencia al interior de algo, se la piensa, en cambio, como una irregularidad de intensidades. Para Deleuze esta diferencia cuantitativa de intensidades surge a partir de otra: entre las fuerzas "activas" y "reactivas" que dan lugar a valores, acontecimientos y cuerpos. A su vez, estas fuerzas activas y reactivas están constituidas por las cualidades "afirmativas" y "negativas" de la voluntad de poder. Asimismo, la voluntad de poder es anterior a las fuerzas que, a su vez, son anteriores al cuerpo (Lash, 1997, p.90). El cuerpo es un objeto 
de competencia entre las fuerzas activas del deseo y las reactivas que puestas en movimiento por el capital se traban en lucha (Lash, 1997, p.92).

El deseo en Deleuze es diferente al conceptualizado por el psicoanálisis que lo piensa como aquello que se instaura por efecto de una falta mítica y constitutiva de la psiquis. En cambio, para Deleuze y Guattari el deseo no supone una ausencia o falta sino que es producción y consiste en flujos de energía, por esto los autores hacen referencia a la máquina deseante.

Ahora bien: ipor qué sostenemos que los aportes de Deleuze y Guattari aquí desarrollados contribuyen a comprender el cuerpo desde una perspectiva posmoderna?

Estaríamos frente a un cuerpo que no está capturado del todo por las instituciones y el discurso. Esta perspectiva se condice con expresiones usuales tales como el vaciamiento de las instituciones y la crisis de las mismas que dieron lugar a numerosas reformas administrativogubernamentales que, más que fortalecerlas, las fueron debilitando y vaciando paulatinamente en un sentido funcional y material. Como señalamos, hacer un trato discursivo del cuerpo implica su pasividad, además es asible a una unidad que le otorgan los discursos modernos (la pedagogía, el médico-psiquiátrico, el psicoanálisis, el jurídico, civil) y la noción de estructura.

La empresa pos-estructuralista propuesta por Deleuze y Guattari apunta a una ruptura de la unidad que se le intenta dar al sujeto del inconciente, ya que su noción des-edipizada del obrar construye resistencias a las prácticas psiquiátricas institucionales; su inconciente des-familiarizado es, al mismo tiempo, un ataque al falocentrismo. Lash plantea que este es un proyecto de la "contra-memoria", pues en la modernidad el discurso crea memoria (Lash, 1997, p.93).

Si pensamos que las instituciones producen a los sujetos al mismo tiempo que estos las reproducen y crean (Castoriadis, 1983), entonces se observarán en paralelo transformaciones en la idea de cuerpo y en la instituciones. Deleuze (1999), basándose en Foucault, se refiere a las instituciones modernas como centros de encierro y sociedades disciplinarias, y a las posmodernas como sociedades de control. El autor plantea que mientras que las primeras utilizan un lenguaje analógico, las segundas emplean uno numérico. Los lugares de encierro constituyen una cuadrícula que ubica la posición del sujeto; en este sentido son moldes, mientras que los controles constituyen una modulación. Las sociedades disciplinarias presentan dos polos: la marca que identifica al individuo y la matrícula que indica su posición en la masa. Plantea Deleuze que ya no estamos frente al par "individuo-masa", dado que los individuos han devenido "dividuales" - un número en una base de datos- y las masas se han convertido en indicadores, datos, mercados o bancos (Deleuze, 1999, p.281).

Se observan también cambios en las estrategias de resistencia estrechamente ligadas con la transformación en el espacio. Negri y Hardt (2002) plantean que en la transición contemporánea al Imperio, los túneles estructurados del topo -analogía planteada por Marx 
para explicar las luchas proletarias: salen en los momentos de conflicto abierto y luego excavan túneles que sería el momento programático de la lucha- han sido reemplazados por las ondulaciones infinitas de la serpiente, idea que se relaciona con la modulación propuesta por Deleuze. En la posmodernidad, las profundidades del mundo moderno y sus pasadizos se han vuelto superficiales. Las luchas actuales se deslizan silenciosamente por la superficie y simplemente concentran sus fuerzas en las articulaciones más elevadas del orden imperial, deben golpear en su corazón y fortaleza (Negri y Hardt, 2002, pp.68 y 69). Los autores sostienen que las luchas serpentinas que presenciamos en la actualidad no manifiestan una táctica revolucionaria clara, por lo que son incomprensibles desde el punto de vista programático (Negri y Hardt, 2002, p.68).

En síntesis, la noción de cuerpo rastreada por Foucault -atravesado por saberes, disciplinas y discursos que lo controlan, identifican y ubican en una posición, dándole unidad- no responde a una idea posmoderna del mismo. Esto es, un cuerpo que no se puede ubicar ni significar claramente desde una posición social sino que se define en las intensidades que están en la superficie. Mientras en la estrategia del "topo" existe una lógica de lo profundo, la estrategia de la "serpiente" se da en los pliegues de la superficie. Estas cuestiones dan cuenta de una transformación en la construcción del espacio: de moldes se pasa a las modulaciones, es decir, de las instituciones de encierro basadas en el modelo Panóptico de control se pasa a la conformación de bases de datos confiables y a las autopistas informáticas (Bauman, 1999, p.69); de la lógica de lo profundo a la de la superficie (Deleuze y Guattari, 1988). Se observan estas transformaciones también en la temporalidad: de ser un tiempo reglado y ordenado propio de las instituciones disciplinarias, con horarios y un control sobre el mismo, se pasa a un tiempo flexible y subjetivo. Por ejemplo, en el ámbito laboral, en la actualidad, existe un mayor interés por la realización de proyectos que por el cumplimiento de un horario; en las escuelas se tiende a poner un mayor énfasis en los procesos personales de aprendizaje que en las pautas normalizadoras.

Por su parte, Deleuze propone un cuerpo no orgánico que tampoco se inscribe en el orden simbólico, sino que es un cuerpo que se define en la diferencia de intensidades presentes en la superficie, en la competencia entre fuerzas activas y reactivas. Distinciones cuantitativas que se van definiendo mutuamente. En el marco de estas intensidades y de un deseo que es potencia y producción (y no ausencia) es que se puede perfilar en Deleuze un cuerpo que emprende resistencias.

\section{LAS TRANSFORMACIONES EN LA FIGURA ESTATAL Y SUS FORMAS JURÍDICAS}

Nos interesa rastrear las transformaciones en la figura estatal en tanto expresión de una particular "tecnología de poder". En este sentido, Foucault plantea estudiar los nexos entre la racionalización y el poder, es decir, el tipo de racionalidad que opera en el ejercicio de los distintos poderes: el pastoral y el político. No se trata de una "razón general" sino un tipo muy específico de racionalidad. Plantea: 
"Pienso en el desarrollo de las técnicas de poder orientadas hacia los individuos y destinadas a dirigirlos de manera continua y permanente. Si el Estado es la forma política de un poder centralizado y centralizador, llamaremos pastoría al poder individualizador (...) intentaré mostrar cómo esa pastoría se vio asociada a su contrario, El Estado". (Foucault, 1989, p.42)

La noción de poder pastoral construida por Foucault se caracteriza por tres cuestiones: el pastor ejerce el poder sobre un rebaño más que sobre un territorio; lo reúne, guía y conduce y, finalmente, la función del pastor es asegurar su salvación (Foucault, 1989, p.45). Asimismo, el poder pastoral exige atención individual de cada miembro del rebaño, de aquí su poder individualizador. De estas características nos interesa destacar que el rebaño existe por la presencia inmediata del pastor, por lo que si este desaparece se dispersa el rebaño (Foucault, 1989, p.45). Esto da cuenta de una manera particular de organizar y ejercer el poder: con presencia inmediata y acción directa e individualizada. Ya hicimos referencia sobre estos aspectos cuando planteamos que en el período clásico hay una inscripción física directa sobre el cuerpo.

Ahora bien, el problema político se plantea en la relación entre el individuo y la multitud. El poder político - poder pastoral laico- opera en el seno del Estado en tanto marco jurídico de la unidad. La racionalidad del poder de Estado es formulada a partir de dos cuerpos doctrinales: la razón de Estado y la teoría de policía (Foucault, 1989, p.61). Estos hitos que caracterizan al Estado moderno sufren transformaciones en la posmodernidad o, según Negri y Hardt (2002), en la lógica del Imperio.

La razón de Estado es considerada como un "arte", es decir, como cierta técnica que se rige por reglas. Por esto el arte de gobernar es racional, no sigue leyes divinas, naturales o humanas. El designio del arte de gobernar es justamente el no reforzar el poder que puede ejercer un príncipe sobre sus súbditos. Su objetivo es reforzar al Estado mismo, es un gobierno que busca incrementar la fuerza del Estado en un marco extensivo y competitivo (Foucault, 1989, pp.63-64). En este punto intentaremos señalar las diferencias con las maneras extensivas del Imperio de Negri y Hardt.

Existe una relación estrecha entre el poder pastoral y el poder en las disciplinas, este último realiza una doble operación: es al mismo tiempo masificador e individualizante, es decir, identifica al individuo al mismo tiempo que lo masifica. Foucault encuentra el origen de este doble objetivo en el poder pastoral del sacerdote-el rebaño y cada una de las ovejas-. Por su parte, el poder civil, a través de otros medios, se ha convertido en un "pastor" laico (Deleuze, 1999, p.281).

Negri y Hardt plantean que la problemática del Estado está determinada en primera instancia por un hecho simple: que haya un orden mundial que se expresa como una formación jurídica. La tarea que se proponen es comprender la constitución del orden que se está formando hoy ${ }^{3}$.

3. Desde el inicio estos autores pretenden separarse de dos maneras de explicar este orden. De los que piensan a la globalización como algo dado y sostienen que este orden surge espontáneamente de las fuerzas globales. La otra 
Sostienen que hay nuevas lógicas que se construyen en torno al imperio, dicen: "... la soberanía imperial marca un cambio de paradigma" (Negri y Hardt, 2002, p.25). En este punto se diferencian de quienes piensan que las tendencias contemporáneas a la formación del imperio no representarían un fenómeno fundamentalmente nuevo, sino simplemente un perfeccionamiento del antiguo imperialismo. Pues los Estados-nación capitalistas continúan ejerciendo una dominación imperialista sobre las demás naciones.

Los autores plantean: "prestar atención al prolongado proceso de transición que condujo desde el derecho soberano de los Estados-nación a las primeras figuras globales del derecho imperial" (Negri y Hart, 2002: 20). Les interesa la transformación jurídica dado que funciona como un síntoma de las modificaciones de la constitución material biopolítica de nuestras sociedades. Estos cambios corresponden no solo a la ley y a las relaciones internacionales sino que las transformaciones jurídicas efectivamente indican los cambios producidos en la constitución material del poder y el orden mundiales (Negri y Hardt, 2002, p.26).

La Organización de las Naciones Unidas - ONU funciona como bisagra en la genealogía de las estructuras jurídicas, desde las internacionales hacia las globales. Por un lado, toda la estructura conceptual de la ONU se basa en el reconocimiento y la legitimación de la soberanía de los Estados individuales y, por consiguiente, se ubica dentro del antiguo esquema de derecho internacional definido por pactos y tratados. Sin embargo, por otro lado, este proceso de legitimación solo es efectivo en la medida en que transfiera el derecho soberano a un centro supranacional real (2002, p.22). En este sentido, se puede hablar de una soberanía supranacional cuya fuente de legitimidad y su ejercicio ponen de manifiesto una serie de problemas políticos, culturales y ontológicos (2002, p.27).

Los procesos de globalización funcionan como una fuente de definiciones jurídicas que tienden a proyectar una única figura supranacional de poder político, observándose cambios en las relaciones globales de poder. Lo que solía ser un conflicto o una competencia entre varias potencias imperialistas ha sido reemplazado por la idea de un único poder que ultra-determina a todas las potencias y las estructuras de forma unitaria. Este es el punto de partida para el estudio del imperio, plantear una nueva noción de derecho o, más bien, de la inscripción de autoridad y un nuevo diseño de la producción de normas e instrumentos legales de coerción que garantizan los contratos y resuelven los conflictos (2002: 26). Dicen:

"En suma, el cambio de paradigma se define, al menos inicialmente, mediante el reconocimiento de que sólo un poder establecido ultra-determinado y relativamente autónomo respecto de los Estados-nación soberanos puede funcionar como el centro del nuevo orden mundial, ejerciendo sobre él una regulación efectiva y, cuando es necesario, la coerción". (Negri y Hardt, 2002, p.30)

visión de la que se separan Hardt y Negri es aquella que plantea que quien dicta el orden es un poder único y centro de la racionalidad que trasciende a las mismas fuerzas globales. 
Este nuevo paradigma funciona en términos positivos. Es tanto sistema como jerarquía, una construcción centralizada de normas y una extendida producción de legitimidad, difundida a lo largo y a lo ancho del espacio mundial. Se configuró como una estructura sistémica, dinámica y flexible que se articula horizontalmente. Se suele aludir al "ejercicio de la autoridad sin gobierno" para indicar la lógica estructural, a veces imperceptible, pero siempre progresivamente efectiva, que arrastra a todos los actores dentro del orden del todo.

La excepcionalidad y la posibilidad de intervención caracterizan tanto al derecho nacional como supranacional. Es decir, poder penetrar excepcionalmente en la ley nacional de los Estados con la intención de prevenir o resolver problemas humanitarios, y a reconfigurarla. El derecho supranacional sobredetermina decisivamente el derecho doméstico. El síntoma más significativo de la transformación es el derecho a la intervención, que es una forma de derecho que, en realidad, es un derecho de policía. Es la formación de un nuevo derecho que se inscribe en el despliegue de la prevención, la represión y la fuerza retórica destinadas a reconstruir el equilibrio social, todas características propias de la función policial.

En síntesis, mientras en la modernidad planteamos que la función de policía otorga mayor fuerza al Estado, en la posmodernidad el derecho de intervención refuerza y legitima al poder ultradeterminado y supranacional, autónomo de los Estados-nación, ubicado en la figura del Imperio.

\section{LOS MECANISMOS DE INCLUSIÓN Y EXCLUSIÓN}

Para el desarrollo de este apartado tendremos en cuenta, por un lado, los aportes de Luhmann (1998) que ayudan a comprender la dinámica de la inclusión-exclusión en el sistema social; por el otro, a Robert Castel y otros autores franceses (Autes, 2004) que ofrecen categorías para pensar el proceso de "desenganche" social y subjetivo. Mientras el primer autor piensa la exclusión en términos dinámicos y sistémicos; los segundos se detienen en los procesos de desafiliación social y de deterioro de la subjetividad.

Luhmann plantea a la inclusión-exclusión como distinción sistémica. La diferenciación implica la formación de sistemas dentro de sistemas (Luhmann, 1998, p.171). Teniendo en cuenta al sistema de la sociedad, depende de la forma de diferenciación que adopte una sociedad para estructurar su división primaria, que es el punto de partida para la inclusión/exclusión (Luhmann, 1998, p.172). La forma de diferenciación de la modernidad es la funcional, en cambio, en la sociedad moderna - para nuestro ensayo la posmodernidad ${ }^{4}$ - las relaciones entre los sistemas funcionales fluctúan y ya no pueden ser determinados para el conjunto de la sociedad.

4. A pesar de que Luhmann no habla de posmodernidad, las fluctuaciones que describe en la forma de diferenciación funcional de la modernidad se condicen con las transformaciones que estamos pensando y desarrollando para la posmodernidad. 
Luhmann plantea que las teorías de la modernización confían en la capacidad de realización de los sistemas funcionales; es decir, esperan que puedan realizarse de forma universal, en poco tiempo y que, al mismo tiempo, garanticen el desarrollo económico, la democratización política, la positivización e implementación del derecho y la intensificación de la investigación científica. Se supone así que los sistemas funcionales concretos pueden encaminarse hacia la modernización apoyándose mutuamente y dando lugar a una regulación uniforme de la inclusión. No obstante, los hechos ecológicos y demográficos muestran serias dudas sobre la viabilidad de este proyecto (Luhmann, 1998, p.181).

En la modernidad es la inclusión el principio de diferenciación de la sociedad, pues en el orden de las relaciones entre inclusión y exclusión sigue confiándose a dispositivos especiales que permiten considerar esta diferencia como interna a la sociedad. La política de la reinserción reclama para sus objetivos al individuo mismo (Luhmann, 1998, p.175). En cambio, en la posmodernidad, y dado que la inclusión en los sistemas funcionales ya no determina cómo y cuán intensamente se toma parte en los otros sistemas funcionales -por ejemplo: qué derechos se tienen y cómo se regula el acceso a los mismos-, el resultado es un considerable relajamiento de la integración en el ámbito de la inclusión (Luhman, 1998, p.190).

En el ámbito de la exclusión se encuentra el cuadro opuesto. En este caso la sociedad está muy integrada porque la exclusión de un sistema funcional implica casi automáticamente la exclusión de otros (Luhmann, 1998: 190). Por ejemplo, si no se accede a un empleo formal tampoco se tiene cobertura médica ni social. La forma de diferenciación funcional -que organizaba la inclusión- no puede ordenar el ámbito de la exclusión, pues esta entra en contradicción con los hechos de la exclusión. Es decir, si bien la base con la que se comprende la lógica de la diferenciación funcional, que es societal-universal, también abarca la exclusión, dado que el derecho es para todos, y la posibilidad de casarse y formar una familia no se le niega a nadie; los hechos de la exclusión y la discriminación ponen de manifiesto su improbabilidad y artificialidad. Sus códigos valen y no valen para la misma sociedad. Por esto, es posible inferir que la distinción entre inclusión (laxamente integrada) y exclusión (integrada consistentemente) es un "supercódigo" con el qué orientarse, si se quiere entender a las sociedades contemporáneas (Luhmann, 1998, p.191).

Luhmann señala una serie de preguntas muy interesantes que muestran cómo entra en contradicción la lógica de diferenciación funcional en el ámbito de la exclusión, dice: ¿Qué significa para el sistema político el que en un número cada vez mayor de zonas pueda asegurarse ya la paz? ¿Qué significa para el sistema jurídico que el ámbito de la exclusión y con él también la policía -como organización vinculadora de la inclusión y la exclusión- y finalmente la política misma pierdan su vinculación con los principios del Estado de derecho, de manera que puede actuarse igual de bien y exitosamente haciéndolo de acuerdo con la ley que en contra de ella? ¿Qué significa para el sistema económico que gran parte de la población esté excluida de los mercados? (Luhmann, 1998, p.194). 
El autor sostiene que estas contradicciones no llevarán al colapso universal de los sistemas funcionales dado que su autopoiesis ${ }^{5}$ es robusta. Es más, si se presta atención a los desarrollos estructurales de estos sistemas, se observa una creciente solidez y prevalencia de la diferencia entre inclusión y exclusión.

En otras palabras, no estamos frente a lógicas "caóticas" sino que el principio de diferenciación se ordena desde la exclusión, por lo que se esperan efectos más expulsivos que de inclusión. Algunos teóricos suelen usar la metáfora del "lavarropas" para ilustrar el mecanismo de la exclusión en la posmodernidad, dado que la fuerza centrípeta expulsa al sujeto al vacío, no habiendo trama simbólica ni institucional que capture al excluido con la intención de reinsertarlo, tal como sucede en la modernidad. La imagen que se utiliza para la modernidad es la de una cuadrícula donde tanto el incluido como el excluido tienen un lugar y posición en la sociedad, aunque habiten instituciones distintas. Los primeros circulan por la escuela y la fábrica, los segundos por manicomios, cárceles y sociedades de fomento.

Sostenemos que Luhmann estaría de acuerdo, al igual que Deleuze, en plantear que estamos frente a un cuerpo real y no simbólico, que implica que la sociedad adquiera otra forma. En esta ocasión hacemos referencia al aspecto real del cuerpo, no en el sentido de fuerza para resistir, tal como se planteó en el primer apartado, sino en el sentido material o biológico del término. Queda pendiente pensar cómo este cuerpo real se puede transformar en un cuerpo activo con capacidad para resistir, cuestión que se esboza al final de las conclusiones.

Luhmann sostiene que en el ámbito de la exclusión los seres humanos ya no son considerados personas sino cuerpos. A modo de ejemplo, si se pasea por una ciudad brasileña, por sus plazas y calles, se necesitará una observación permanente de la postura, separación y aglomeración de cuerpos para conocer la competencia social básica. Dice: "El desplazamiento de la atención y la relevancia comunicativa desde la persona hacia el cuerpo no significa que la sociedad se suspenda, sino sólo que ésta adopta otra forma" (Luhmann, 1998, p.194). La fuerza física, la sexualidad, la satisfacción de las necesidades primarias quedan de nuevo liberadas (dejan de atender a aspectos simbólicos), lo que impide el desarrollo de una comunicación rica en presupuestos (Luhmann, 1998, p.195). Precisamente cuando el otro cuenta como cuerpo, es cuando mayor es el peligro para el cuerpo y la vida propios (Luhmann, 1998, p.194).

5. Los sistemas autopoiéticos tienden a sostenerse y reproducirse a sí mismos. Cuando hay influencias del entorno sobre el sistema (por ejemplo, que una crisis económica produzca daño en el sistema social y que este provoque trastornos en el sistema político), no las debemos considerar como si fueran influencias directas sino como influencias que eventualmente pueden ser resueltas dentro del sistema donde tuvieron su origen, es decir, sin que trasciendan el medio sistémico de inicio. Ahora bien, esta situación cambia cuando rebasan las fronteras sistémicas. 
El incremento de la pobreza en la ciudad de Buenos Aires a inicios del siglo XXI, luego de una fuerte crisis ocurrida en el año $2001^{6}$, desafortunadamente ilustra esta lúcida consideración de Luhmann. Esta situación generó claramente un cambio en la urbanidad de la ciudad y en ese momento se observaban cada vez más personas indigentes "viviendo" en las calles de Buenos Aires. También se podían ver a los "cartoneros" (aún hoy día subsisten los cartoneros) quienes remueven en las bolsas de basura de las calles porteñas para conseguir cartones para su comercialización. Si bien tanto el caso del indigente como el del cartonero tienen sus particularidades, dado que el segundo ejemplo es percibido como "trabajo" en contexto de crisis, lo que tienen en común es que estas situaciones orillan a la persona a que pierda paulatinamente su condición de persona y su dignidad, para pasar a adquirir fuerza el cuerpo real, anudado a la satisfacción de necesidades básicas.

Entonces, como se planteó al inicio del artículo, existe una relación entre una sociedad que se integra a partir de la exclusión (Luhmann, 1998) y la prevalencia del sentido real del cuerpo. Por esto, el cuerpo pasivo y simbólico de Foucault no resulta una noción útil para comprender la lógica de diferenciación de la posmodernidad. Asimismo, esta idea es coherente con una figura supranacional del poder político que define a una soberanía no ya sustentada en los Estado-nación sino en lo supranacional. Es decir, se trata de una soberanía volátil y poco asible, que le permite al Estado desentenderse de los problemas sociales a la vista. Pues, ante una situación de extrema pobreza iquién es el responsable y a quién se tiene que reclamar?

Para finalizar este apartado, aludiremos a algunos aportes de Robert Castel y otros autores (Autes, 2004) sobre el tema de la exclusión. Estos autores, a diferencia de Luhmann, para su análisis tienen en cuenta aspectos subjetivos y sociales. Para sus trabajos consideran los relatos de sujetos concretos e historias de vida. De estas narraciones se desprende que el sujeto "desenganchado" no se encuentra excluido solo de lo social sino de sí mismo, pues la exclusión implica el inicio de un proceso de desmoronamiento subjetivo. Se alude a este proceso a través de nociones tales como desafiliación, desenganche y renunciamiento. Castel, quien realiza una reconstrucción histórica del trabajo asalariado, plantea una doble desafiliación: de la condición laboral y del lazo social, "elabora su concepto de desafiliación mostrando que no se trata solo de una ruptura con el salario, sino además con lo que llama "lazo social", pues se observa una pérdida del sentido de pertenencia de los individuos" (Autes, 2004, p.30). La falta de empleo, combinado con ausencia de políticas que compensen los efectos de la flexibilidad laboral, tiene por consecuencia -sobre todo en sujetos no insertos en redes formales o informales- el inicio de un proceso de desafiliación que suele conllevar a un deterioro de la subjetividad: "El individuo renuncia a volver a encontrar trabajo, siente que no es como los demás" (Autes, 2004, p.27).

6. En Argentina, en diciembre de 2001, hubo una importante crisis económica y política que llevó a la renuncia del presidente Fernando de la Rúa. Es en este contexto que aparecen las asambleas barriales conformadas por vecinos de distintos barrios. Las asambleas adquirieron mayor protagonismo en las principales ciudades del país, principalmente en Buenos Aires. 
Ahora bien ipor qué consideramos que es importante poder establecer distintos niveles o matices de la pobreza? Los autores establecen distintas etapas en el proceso de exclusión, por ejemplo, pérdida de trabajo, luego el desenganche que implica la renuncia a volver a buscar trabajo y, finalmente, desmoronamiento subjetivo. Castel define distintas zonas: la de integración, vulnerabilidad (definida por la precariedad laboral, también por la pérdida de trabajo, pero manteniendo las redes informales que le pueden permitir al individuo volver a insertarse) y exclusión. Castel también diferencia entre el pobre legítimo, el ilegítimo y el pobre socorrido. Consideramos que es fundamental poder establecer los gradientes y matices de la pobreza para el diseño de políticas públicas en esta problemática. Asimismo, es importante apuntar a que el sujeto desafiliado realice una reflexión sobre sus capacidades y evalúe sus posibilidades para volver a integrarse laboral y socialmente ya sea de modo formal o informal.

\section{CONCLUSIONES}

Teniendo en cuenta los procesos de transformación entre la modernidad y la posmodernidad, se mostró que el cuerpo de Foucault mediado por saberes e instituciones se condice con un poder político territorializado -los Estados-nación- que cuenta con dispositivos complejos para vigilar, disciplinar y corregir a los sujetos. Se relaciona también con un principio de diferenciación regido por la inclusión, por lo que se busca reinsertar a los desviados de la norma, creando instituciones para ello. El cuerpo de Foucault se vuelve pasivo frente a la perspectiva de la posmodernidad, por esto rescatamos la noción de cuerpo de Deleuze, que se define en las diferencias entre intensidades de las fuerzas activas y reactivas. En este artículo hemos pensado el aspecto real del cuerpo en dos sentidos, por un lado, en tanto materialidad evidente en las calles, en lo urbano con un soporte simbólico deteriorado y, por el otro, como cuerpo activo que resiste.

En una sociedad "integrada" bajo el principio de la exclusión, con fuerte retraimiento del Estado y, al mismo tiempo, con la imposición de la lógica del imperio, única, supranacional y ultradeterminada, autónoma de los Estados-nación, a los que se puede intervenir; frente a esta situación se impulsan procesos sociales que llevan a la desafiliación de los sujetos. La descripción que hace Luhmann sobre la prevalencia de los cuerpos en la ciudad brasilera, nos muestra cuerpos reales que han perdido soporte simbólico entre otros del Estado, pues son "marginales en general", no se establecen distinciones entre ellos tal como sucedía en la modernidad; es así como en las calles urbanas pueden convivir un loco, un desempleado y un indigente.

Ahora bien, para finalizar el artículo retomamos una pregunta pendiente ¿Cómo este cuerpo real se puede transformar en uno activo, con capacidad para resistir? La respuesta está en la acción colectiva y en la trama simbólica e identitaria que se puede restituir frente a la posibilidad de accionar con otros. El participar de un movimiento social habilita al sujeto a pensarse ante sí y los otros de manera diferente, volviendo a tener dignidad. Un ejemplo de esta posibilidad de restitución subjetiva es el Movimiento de Trabajadores Desocupados MTD, en la Argentina. El hecho de poder participar colectivamente de acciones concretas y de 
un proyecto compartido genera potencialidades "salvadoras" y restitutivas de la subjetividad (Svampa y Pereyra, 2003). Se despierta la capacidad de imaginación fundamental para instaurar nuevas prácticas y para sostener una utopía que otorgue un nuevo sentido a la vida de las personas (Castoriadis, 1999).

\section{REFERENCIAS}

- $\quad$ Autes, M. (2004). Tres formas de desligadura. En Karsz, S. (coord.). La exclusión: bordeando sus fronteras. Gedisa: Barcelona.

- Alexander, J.C. et al. (comps.) (1994). El vínculo macro-micro. México: Universidad de Guadalajara.

- Baumann, Z. (1999). Guerras por el espacio: informe de una carrera. En La globalización. Consecuencias humanas. Buenos Aires: Fondo de Cultura Económica.

- Castoriadis, C. (1983). La institución imaginaria y lo imaginario, primera aproximación. Tomo I: La institución imaginaria de la sociedad. Barcelona: Tusquets.

- Castoriadis, C. (1999). Imaginario e imaginación en la encrucijada. En Figuras de lo pensable. Madrid: Editorial Frónesis.

- Deleuze, G. (1999). Post-scriptum sobre las sociedades de control. En Conversaciones, Valencia: Pre-textos.

- $\quad$ Deleuze, G. y Guattari, F. (1988). Rizoma. En Mil Mesetas, Valencia: Pre-Textos.

- Falleti, V. (2012). Movilización y protesta de las clases medias argentinas. Cacerolazo y asambleas barriales. México: UAM Xochimilco, CEIICH-UNAM, CLACSO.

- Foucault, M. (1976). El cuerpo de los condenados, Parte Suplicio. En Vigiliar y Castigar, Buenos Aires: Siglo XXI.

- Foucault, M. (1976). Los cuerpos dóciles, Parte Disciplina. En Vigiliar y Castigar, Buenos Aires: Siglo XXI.

- Foucault, M. (1989). Hacia una crítica de la razón política. En Marquet Montiel, A. (edit.). El poder: cuatro conferencias. México: UAM-Azcapotzalco.

- Lash, S. (1997). Posmodernismo: hacia una exposición sociológica. En Sociología de la Posmodernidad. Buenos Aires: Amorrortu. 
- Lash, S. (1997). La genealogía y el cuerpo: Foucault, Deleuze y Nietzsche. En Sociología de la Posmodernidad. Buenos Aires: Amorrortu.

- Lash, S. (1997). Posmodernidad y deseo. En Sociología de la Posmodernidad. Buenos Aires: Amorrortu.

- Luhmann, N. (1998) Inclusión y Exclusión. En Complejidad y modernidad. De la unidad a la diferencia. Madrid: Trotta.

- Negri, A. \& Hardt, M. (2002). Imperio. Buenos Aires: Paidós.

- Svampa, M. \& Pereyra, S. (2003). Entre la ruta y el barrio. La experiencia de organizaciones piqueteras. Buenos Aires: Editorial Biblos. 